AIP Applied Physics

\title{
New magnetic configuration in paramagnetic phase of $\mathrm{HoCo} 2$
}

C. M. Bonilla, I. Calvo, J. Herrero-Albillos, A. I. Figueroa, C. Castan-Guerrero et al.

Citation: J. Appl. Phys. 111, $07 \mathrm{E} 315$ (2012); doi: 10.1063/1.3672258

View online: http://dx.doi.org/10.1063/1.3672258

View Table of Contents: http://jap.aip.org/resource/1/JAPIAU/v111/i7

Published by the American Institute of Physics.

\section{Related Articles}

Controlling the current flux in magnetic-barrier induced graphene waveguide Appl. Phys. Lett. 101, 163104 (2012)

Hard magnetic properties of Mn-Ga melt-spun ribbons

J. Appl. Phys. 112, 083901 (2012)

Influence of chemical pressure in Sn-substituted Ni2MnGa Heusler alloy: Experimental and theoretical studies J. Appl. Phys. 112, 073921 (2012)

Hysteresis effects in the inverse magnetocaloric effect in martensitic Ni-Mn-In and Ni-Mn-Sn

J. Appl. Phys. 112, 073914 (2012)

Magnetic and electronic properties of $\mathrm{Fe}$ and $\mathrm{Ni}$ codoped $\mathrm{SnO} 2$

J. Appl. Phys. 112, 073917 (2012)

\section{Additional information on J. Appl. Phys.}

Journal Homepage: http://jap.aip.org/

Journal Information: http://jap.aip.org/about/about_the_journal

Top downloads: http://jap.aip.org/features/most_downloaded

Information for Authors: http://jap.aip.org/authors

\section{ADVERTISEMENT}

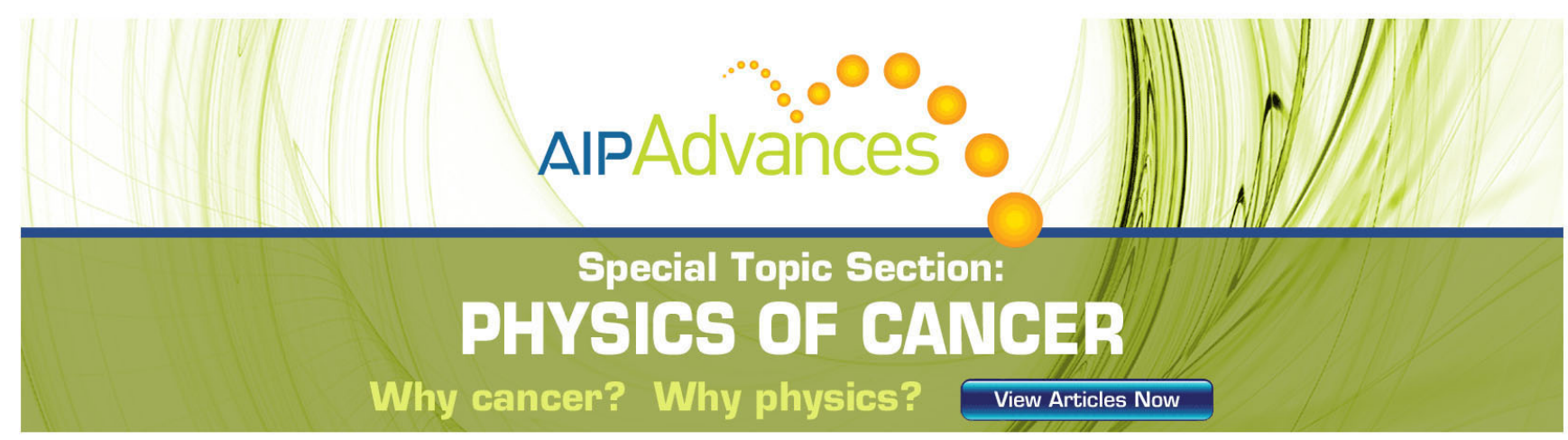




\title{
New magnetic configuration in paramagnetic phase of $\mathrm{HoCo}_{2}$
}

\author{
C. M. Bonilla, ${ }^{1, a)}$ I. Calvo, ${ }^{1,2}$ J. Herrero-Albillos,,${ }^{1,3,4}$ A. I. Figueroa, ${ }^{1}$ C. Castan-Guerrero, ${ }^{1}$ \\ J. Bartolomé, ${ }^{1}$ J. A. Rodriguez-Velamazan, ${ }^{1,2}$ D. Schmitz, ${ }^{4}$ E. Weschke, ${ }^{4}$ D. Paudyal,,${ }^{5,6}$ \\ V. K. Pecharsky, ${ }^{5,6}$ K. A. Gschneidner Jr., ${ }^{5,6}$ F. Bartolomé, ${ }^{1}$ and L. M. García ${ }^{1}$ \\ ${ }^{1}$ Instituto de Ciencia de Materiales de Aragón. CSIC-Universidad de Zaragoza, Departamento de Física de la \\ Materia Condensada, Pedro Cerbuna 12, 50009 Zaragoza, Spain \\ ${ }^{2}$ Institute Laue Langevin, 38042 Grenoble, France \\ ${ }^{3}$ Centro Universitario de la Defensa, Academia General Militar-Uni. Zaragoza, 50090 Zaragoza, Spain \\ ${ }^{4}$ Helmholtz-Zentrum Berlin für Materialien und Energie GmbH, Albert-Einstein-Str. 15, 12489 Berlin, \\ Germany \\ ${ }^{5}$ Ames Laboratory, U.S. Department of Energy, Iowa State University, Ames, Iowa 50011-3020, USA \\ ${ }^{6}$ Department of Materials Science and Engineering, Iowa State University, Ames, Iowa 50011-2300, USA
}

(Presented 31 October 2011; received 23 September 2011; accepted 25 October 2011; published online 23 February 2012)

\begin{abstract}
X-ray magnetic circular dichroism (XMCD) measurements on $\mathrm{HoCo}_{2}$ reveal the inversion of Co moment at temperatures higher than the critical temperature, $T_{c}$, showing that the net magnetization under a field of the Ho and Co sublattices remain antiparallel even above $T_{c}$. The Ho moment also changes its orientation to align antiparallel to the applied field at high temperature giving rise to a new magnetic configuration in the paramagnetic regime. Transverse susceptibility (TS) and small angle neutron scattering (SANS) measurements performed above $T_{c}$ indicate the existence of sizable magnetic short-range correlated regions in $\mathrm{HoCO}_{2}$. First principles calculations based on spin polarized local-density approximation, $\mathrm{LSDA}+\mathrm{U}$ have been performed to obtain insights on the origin of the short-range correlated volume. (C) 2012 American Institute of Physics. [doi:10.1063/1.3672258]
\end{abstract}

\section{INTRODUCTION}

For more than 20 years, the mechanisms that define the magnetism in $R \mathrm{Co}_{2}$ ( $R=$ rare-earth) compounds have been intensively studied as they are an ideal starting point to understand the physical properties of other intermetallic compounds. ${ }^{1-3}$ In magnetic $R \mathrm{Co}_{2}$, the exchange interaction, $J$, between $R 4 f$ from a heavy rare earth and Co $3 d$ is always negative, and one sublattice tends to align antiparallel to the magnetic moment of the other. In the paramagnetic regime, the hybridization of Co $3 d$ and $R 5 d$ is responsible for many physical properties observed in these systems. ${ }^{4}$ However, a new magnetic configuration within the paramagnetic phase of $\mathrm{ErCo}_{2}$ was identified in $2007^{5}$ as result of different experimental techniques. Within this magnetic configuration, named "parimagnetism,", the Co moments are disordered at the long-range but a net Co magnetic moment, antiparallel to the applied field and to Erbium moment, is found. At a certain temperature, denoted as flipping temperature $T_{f}$, well above the critical temperature, $T_{c}$, the Co moment changes its preferred orientation recovering the normal paramagnetic configuration. Short-range order correlations between the Co atoms have been identified within this new magnetic configuration. ${ }^{5}$ We have selected $\mathrm{HoCo}_{2}$ compounds to investigate whether parimagnetism is present in other lanthanide-based $R \mathrm{Co}_{2}$.

Polycrystalline ingots of $\mathrm{HoCo}_{2}$ were prepared following the procedure described in a previous work. ${ }^{6} \mathrm{X}$-ray diffraction (XRD) pattern was recorded in a Rigaku RTO 500RC difractometer using $\mathrm{Cu}-\mathrm{K} \alpha$ radiation. The Rietveld analysis shows no impurities within the 5\% of accuracy of

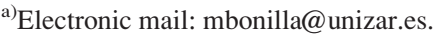

powder diffraction methods. Magnetic characterization as well as the TS measurements were performed at the Servicio de Medidas Físicas of the University of Zaragoza-CSIC. Magnetization as a function of temperature measurements show $T_{c}=76 \mathrm{~K}$.

$\mathrm{X}$-ray absorption (XAS) and x-ray magnetic circular dichroism (XMCD) spectra at the Co $L_{2,3}$ and Ho $M_{5}$ edges were measured at temperatures between $5 \mathrm{~K}$ and $350 \mathrm{~K}$ at $H=1 \mathrm{~T}$ in UE46-PGM1 beamline at the BESSY synchrotron facility. To prevent surface oxidation, samples were cleaved under high vacuum before starting the exposure to the x-rays. Figure 1 shows the XMCD spectra for both Co $L_{2,3}$
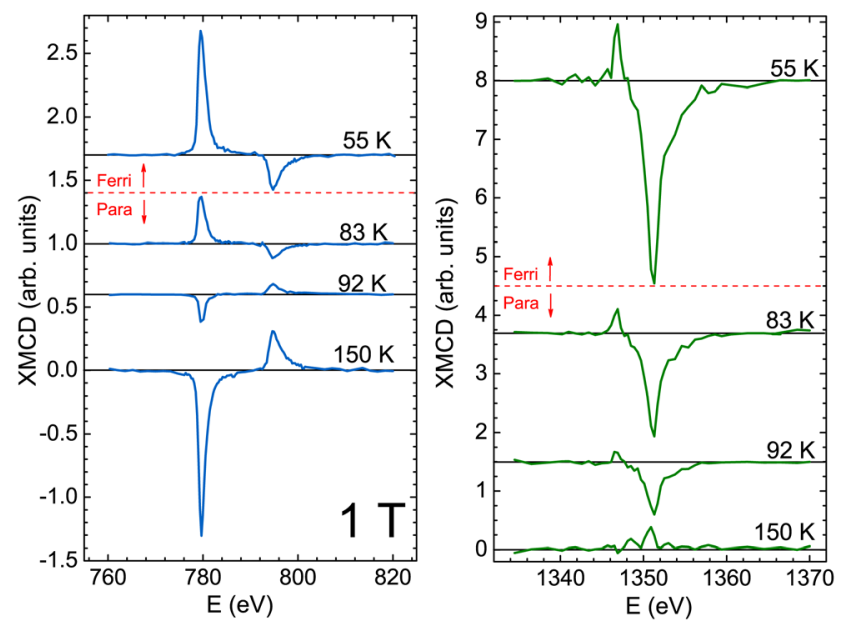

FIG. 1. (Color online) XMCD spectra for selected temperatures at the Co $L_{2,3}$ (left) and Ho $M_{5}$ (right) edges. Measurements were recorded in the compound $\mathrm{HoCo}_{2}$ at $H=1 \mathrm{~T}$. The dashed line separates the spectra measured above and below $T_{c}$. 


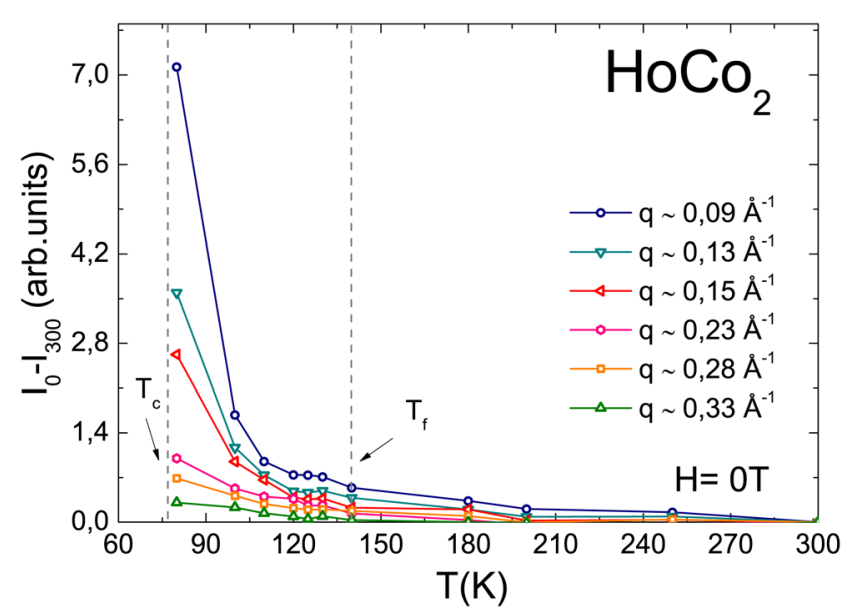

FIG. 2. (Color online) Temperature dependence of $I_{0}-I_{300 \mathrm{~K}}$ at selected $q$ values for $\mathrm{HoCo}_{2}$ at zero applied magnetic field.

(left) and Ho $M_{5}$ (right) edges at selected temperatures. The sign of XMCD signal for Co $L_{2,3}$ changes above $83 \mathrm{~K}$, which implies that the ferrimagnetic coupling between the Co and the Ho moments remains within the paramagnetic state. The parimagnetic alignment observed just above $T_{c}$ has been previously explained in $\mathrm{ErCo}_{2}$ (Ref. 5) as a result of competition between: (i) the Co-Co exchange interactions (which are the strongest interactions in the system), (ii) the exchange field due to the rare-earth sublattice, and (iii) the applied field, $H$. The sign of the Co moment sublattice depends on the temperature and $H$. While the Co-Co exchange interactions align the magnetic moments of Co atoms, the exchange field due to the Ho sublattice tends to align the Co moments antiparallel to the Ho moments. In the parimagnetic configuration, the exchange field from Ho induces an antiparallel arrangement between $\mathrm{Co}$ and Ho net magnetic moments up to a certain flipping temperature $T_{f_{1}}$, which in the case of $\mathrm{HoCo}_{2}$ is between $83 \mathrm{~K}$ and $92 \mathrm{~K}$. Surprisingly as the temperature is raised, the sign of the XMCD signal for Ho $\mathrm{M}_{5}$ also changes at certain temperature $T_{f_{2}}$. The bottom panel on the right side of Fig. 1 shows the inversion of Ho moment at $150 \mathrm{~K}$. This magnetic configuration remains up to $350 \mathrm{~K}$. The measurements show that the Co and Ho sublattice magnetization values are small, but the net moment is different from zero well above $T_{c}$ in both cases. At $T>T_{f_{2}}$, the moment of the Ho sublattice is aligned antiparallel to the moment of Co sublattice and $H$ due to the Co-Co exchange interaction.

The origin of the parimagnetic configuration observed just above $T_{c}$ in $\mathrm{ErCo}_{2}$ has been explained due to the collective flipping of the short-range magnetic correlated regions formed by Co moments. ${ }^{5}$ By means of small angle neutron scattering (SANS) we aimed to observe the occurrence of short-range magnetic correlations in the paramagnetic state of $\mathrm{HoCo}_{2}$. SANS measurements were performed in D16 difractometer at the Institute Laue Langevin. The neutron wavelength used was $\lambda=4.54 \AA$ and the SANS intensity data were collected at zero applied magnetic field and at temperatures between $4 \mathrm{~K}$ and $300 \mathrm{~K}$. From the total SANS intensity, $I_{0}$, only the magnetic intensity, $I_{m}$, is relevant for this study because $I_{m}$ comes from the clustering structures. At $300 \mathrm{~K}$, magnetic correlations are expected to be minor. The difference between $I_{0}$ and the SANS intensity recorded at $300 \mathrm{~K}$ can be analyzed to give a good approximation of the evolution of the correlation length with temperature. Figure 2 shows the temperature dependence $I_{0}-I_{300 \mathrm{~K}}$ for selected $q$ values. The intensity increases as the temperature is reduced to reach a plateau at $140 \mathrm{~K}$, which indicates the occurrence of magnetic correlated regions close to this temperature. Close to $T_{c}$, the curves diverge to infinity as the system approaches to the long-range order.

The spin dynamics of $\mathrm{HoCo}_{2}$ magnetic moments were studied by means of radio-frequency transverse susceptibility (TS). The analysis of the TS profile have previously proved the existence of magnetic clusters in $\mathrm{ErCO}_{2} .{ }^{7}$ The measurements in $\mathrm{HoCO}_{2}$ polycrystalline sample were performed using a self-resonant circuit oscillator based on a simple inverter cell using CMOS transistors in cross-coupled topology. Details of the technique and the circuit can be found in Refs. 7 and 8, respectively. In this particular case, we are interested in the quantity $\left[\Delta \chi_{T} /\right] \%=\left[\chi_{T}(H)-\chi_{T}^{\text {sat }}\right] / \chi_{T}^{\text {sat }} \times 100 \%$ as a function of the applied field $H$, here $\chi_{T}^{\text {sat }}$ is the transverse susceptibility at the saturating field $H^{\text {sat }}$, which in this study has been selected as $1 \mathrm{~T}$. Figure 3 shows the bipolar TS scans for selected temperatures at $10 \mathrm{~K}, 85 \mathrm{~K}, 110 \mathrm{~K}$, and $150 \mathrm{~K}$. The TS profile shows pronounced switching peaks in the ferrimagnetic regime. Close to $T_{c}$, the peaks are narrower as a manifestation of a collective process of Ho moments at the magnetic transition. However, for $T_{c}<T<150 \mathrm{~K}, H_{s}$ values are clearly lower but finite suggesting the occurrence of magnetic clusters in this range of temperatures. The shape of TS profiles above $T_{c}$ coincides with the different magnetic configurations observed by XMCD. As combination of TS measurements and our XMCD study, we propose a scheme of new magnetic configurations for $\mathrm{HoCO}_{2}$ shown in Fig. 4. The Co and Ho magnetization are arranged antiparallel below $T_{c}$. Above $T_{c}$ and up to a certain temperature $T_{f_{1}}$, the Co magnetization sublattice on average is aligned antiparallel to the applied field and to the Ho magnetization. Above $T_{f_{1}}$, the Co magnetization on average is aligned to $H$. At certain
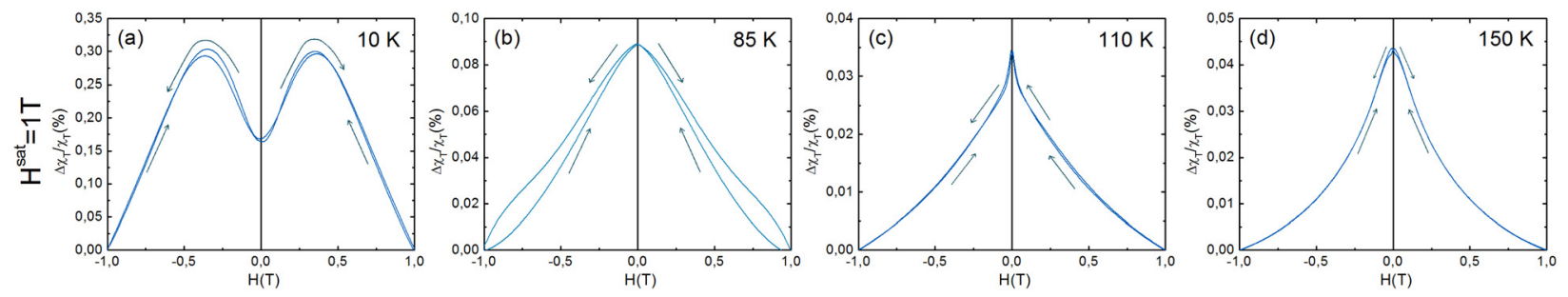

FIG. 3. (Color online) TS scans of $\mathrm{HoCo}_{2}$ for selected fields and temperatures. 


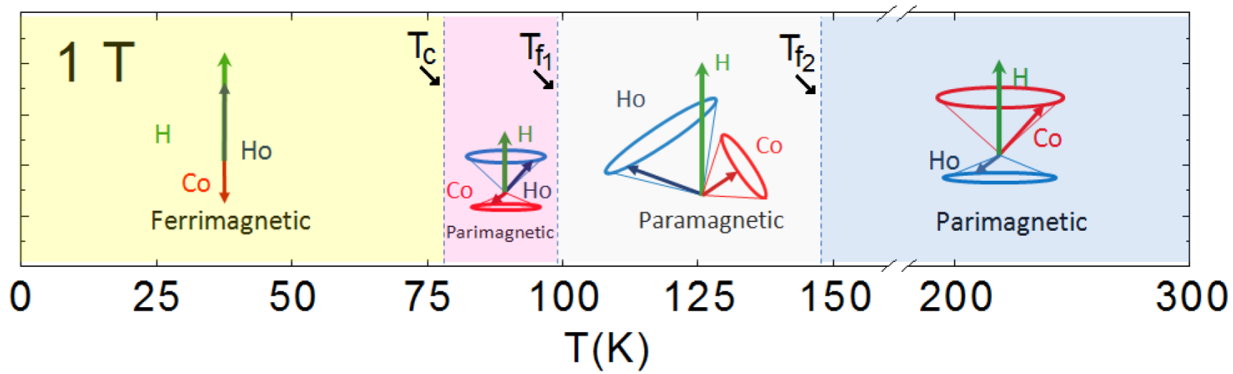

FIG. 4. (Color online) Scheme of magnetic configurations found at different temperatures in the ferrimagnetic and paramagnetic regimes of $\mathrm{HoCo}_{2}$.

temperatures $T_{f_{2}}>T_{f_{1}}$, the Ho magnetization sublattice aligns antiparallel to the applied field and Co moment.

First principles calculations based on the LSDA $+U$ approximation have also been performed for $\mathrm{HoCo}_{2}$ to obtain insights on the origin of the Co-short-range correlated volume. In the theoretical model, we have considered a P1 unit cell with eight inequivalent positions for Ho atoms and 16 inequivalent positions for Co atoms, a $U=4.0 \mathrm{eV}$ value has been used in the calculations. Magnetic moment has been assigned to each atom in such a way that the net magnetic moment in the unit cell is 0 to simulate the paramagnetic state. The inclusion of an impurity located at an interstitial position of the cell is needed to give rise to the nucleation of Co clusters. The imbalance of electron charge density between different Co sites due to the impurity within the unit cell could give rise the formation of magnetic short range correlations between Co atoms.

In summary, our XMCD study demonstrates that Ho and Co net magnetic moments are different from zero within the paramagnetic region of $\mathrm{HoCo}_{2}$. A new magnetic phase diagram proposed for $\mathrm{HoCo}_{2}$ shows two parimagnetic configurations above $T_{c}$. The origin of these new magnetic configurations, is ascribed to the magnetic clusters detected by SANS and TS techniques. Theoretical simulations suggest that an impurity is needed for the nucleation of the magnetic correlated regions in the sample. These results also suggest that parimagnetism may be a common phenomenon among ferrimagnetic $\mathrm{RCo}_{2}$.

\section{ACKNOWLEDGMENTS}

The financial support of MAT2011-23791, and Aragonese IMANA projects, as well as from European FEDER funds is acknowledged. Work at the Ames Laboratory is supported by the US DOE under Contract No. DE- AC02-07CH11358. The authors acknowledge neutron source ILL and photon source BESSY. C.M. Bonilla acknowledges a Spanish MICINN grant.

${ }^{1}$ N. H. Duc, P. E. Brommer, and K. H. J. Buschow, Handbook on Magnetic Materials (Elsevier, Amsterdam, 1999), Vol. 12, p. 259.

${ }^{2}$ H. Yamada, J. Inoue, K. Terao, S. Kanda, and M. Shimizu, J. Phys. F: Met. Phys. 14, 1943 (1984).

${ }^{3}$ D. Bloch, D. M. Edwards, M. Shimizu, and J. Voiron, J. Phys. F: Met. Phys. 5, 1217 (1975).

${ }^{4}$ E. Gratz and A. S. Markosyan, J. Phys.: Condens. Matter 13, R385 (2001).

${ }^{5}$ J. Herrero-Albillos,F. Bartolomé,L. M. García, A. T. Young, T. Funk, J. Campo, and G. J. Cuello, Phys. Rev. B 76, 094409 (2007).

${ }^{6}$ J. Herrero-Albillos, F. Casanova, F. Bartolomé, L. M. García, A. Labarta, and X. Batlle, J. Magn. Magn. Mater. 290-291, 682 (2005).

${ }^{7}$ A. I. Figueroa, S. Chandra, M. H. Phan, H. Srikanth, C. M. Bonilla, F. Bartolomé, L. M. García, J. Bartolomé, and J. Herrero-Albillos, J. Appl. Phys. 109, $07 \mathrm{E} 118$ (2011).

${ }^{8}$ H. Srikanth, J. Wiggins, and H. Rees, Rev. Sci. Instrum. 70, 3097 (1999). 